\title{
A new look
}

\section{Although Nature Photonics may have had a face-lift, its mission to bring you a selection of the most exciting research findings in all areas of optics and optoelectronics remains the same.}

The start of a new year is often a good time to embrace change and revisit the ways things are done, and it's just the same at a journal. With this in mind, you will immediately notice that the January issue of Nature Photonics looks somewhat different. Whether you're opening a print copy of the journal or reading its content online, you will see that our pages have undergone some serious cosmetic surgery. The main aim was to revitalize the design, which we have achieved by updating our fonts, removing unwanted white space and creating a new fresh look that presents information in a clearer, more concise fashion. The result is a journal with a look that is easier to read and navigate.

We've also taken the opportunity to make a few other changes. To bring us in line with other Nature research journals in the physical sciences its time to say goodbye to "This issue" and "Photonics at NPG". At the same time, we've expanded our Technology Focus supplement in 2009 with longer industry perspective pieces, a double-page spread of research highlights and the addition of a profile piece describing the activities of a young, emerging firm in the relevant area. The aim is to provide a more concentrated and in-depth insight into an important technology within photonics that has a strong application and industrial focus. In 2009, we will be running four such
Technology Focus supplements on the topics of semiconductor light sources, materials processing, imaging and organic photonics. The first of these - semiconductor light sources - appears this month and brings together a collection of articles on the topics of quantum cascade lasers (QCLs), long-wavelength vertical-cavity surfaceemitting lasers, quantum-dot lasers and tapered-laser diodes, as well as an update on the business and product news in the sector.

By a fortunate coincidence, several of the primary research findings reported in this issue are also on the topic of semiconductor lasers - in particular QCLs, which operate in the terahertz region. For those that aren't familiar with this region, it lies in the far-infrared and is ripe with potential applications in spectroscopy, imaging and communications. However, its position in the electromagnetic spectrum represents a boundary or gap between photonics and electronics that has caused problems for semiconductor technology. So far, despite some inspiring research, semiconductor technology has not yet been able to offer the kind of convenient, high-performance, mass-producible sources that are required for many applications to become cost effective or practical. But there is good news: the signs that QCLs are making good progress to solving this situation appear in this issue with the report of semiconductor

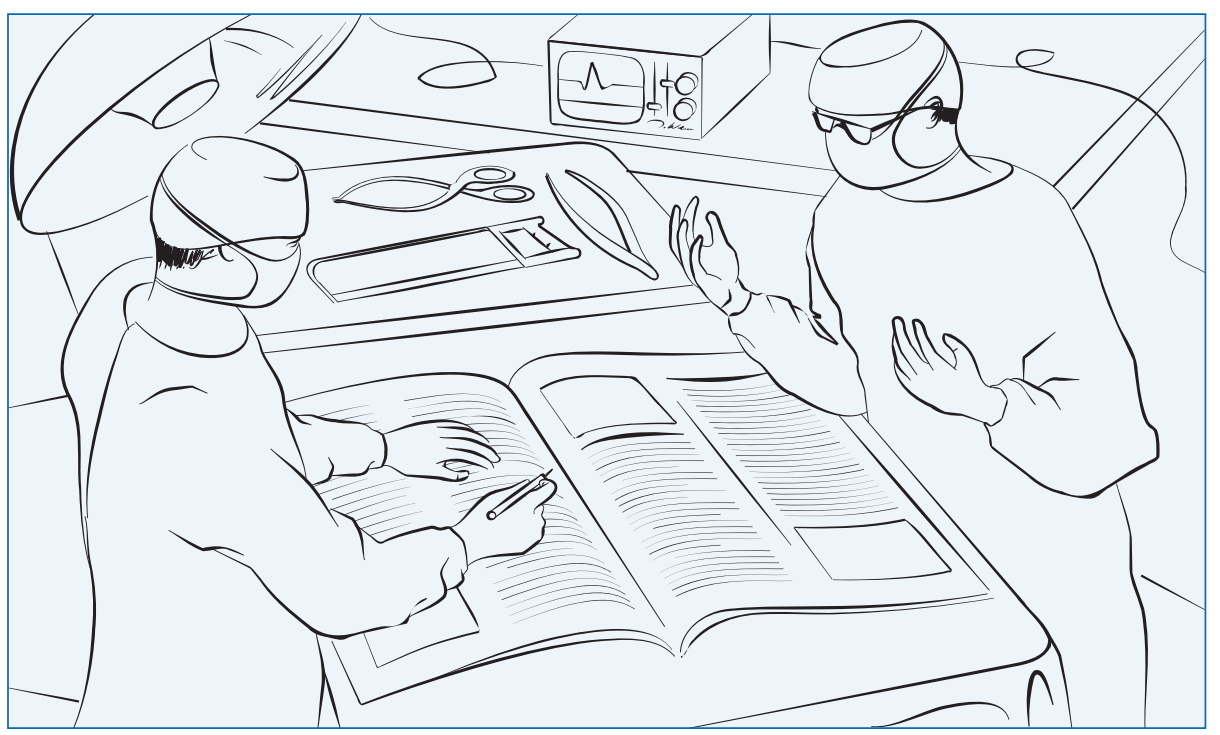

terahertz lasers that operate at record temperatures and new frequencies, new fundamental regimes of operation, and a new top-emitting design that promises better beam-shapes, higher output-powers and the prospect of compact arrays of devices. Although the elevated operatingtemperatures of up to $225 \mathrm{~K}$ are achieved by applying an extreme magnetic field, which of course is not practical in a commercial device, it does clearly demonstrate that other electron-confinement schemes based on structural engineering should reap similar benefits. Indeed, as one of the paper's co-authors in our back-page interview explains, many experts in the area are confident that by 2010 terahertz-QCLs will be operating at $240 \mathrm{~K}$, within the reach of thermoelectric coolers. If this prediction comes true then it really will mark an important milestone for the field, and applications are likely to take off.

\section{Although we look different, our core values remain identical.}

Finally, although the appearance of the journal may look different, we would like to reassure you that our core values reporting research findings of the highest quality and complementing them with accessible articles that are of broad appeal to optics researchers in both academia and industry — remain identical.

As Nature Photonics enters its third year, we believe it's an exciting time for both the journal and for photonics in general. Following the positive feedback on our August 2008 special focus on slow light, we have plans for several more focus issues in 2009, and of course we will continue our programme of regular review articles throughout the year.

We are also busy with preparations for another Nature Photonics conference that will take place in Tokyo on 20-22 October 2009 on future perspectives for photovoltaics. This will be our second event and follows our successful 2007 conference on the future of optical communications. A website for the 2009 event is currently being designed, and we will update you when it's ready and the programme of speakers has been finalized. 